\title{
Existence of the positive solutions for a tripled system of fractional differential equations via integral boundary conditions
}

Hojjat Afshari ${ }^{a}$, Hadi Shojaat ${ }^{\mathrm{b}}$, Mansoureh Siahkali Moradic

${ }^{a}$ Department of Mathematics, Faculty of Sciences, University of Bonab, Bonab, Iran.

${ }^{b}$ Department of Mathematics, Farhangian University, Qazvin, Iran.

${ }^{c}$ Department of Mathematics, Azarbaijan Shahid Madani University, Tabriz, Iran.

\begin{abstract} following tripled system of fractional differential equations.

$$
\left\{\begin{array}{rrr}
D^{\alpha} u(\zeta)+a(\zeta) f(\zeta, v(\zeta), \omega(\zeta))=0, & u(0)=0, & u(1)=\int_{0}^{1} \phi(\zeta) u(\zeta) d \zeta \\
D^{\beta} v(\zeta)+b(\zeta) g(\zeta, u(\zeta), \omega(\zeta))=0, & v(0)=0, & v(1)=\int_{0}^{1} \psi(\zeta) v(\zeta) d \zeta \\
D^{\gamma} \omega(\zeta)+c(\zeta) h(\zeta, u(\zeta), v(\zeta))=0, & \omega(0)=0, & \omega(1)=\int_{0}^{1} \eta(\zeta) \omega(\zeta) d \zeta
\end{array}\right.
$$
\end{abstract}

The purpose of this paper, is studying the existence and nonexistence of positive solutions to a class of a

where $0 \leq \zeta \leq 1,1<\alpha, \beta, \gamma \leq 2, a, b, c \in C((0,1),[0, \infty)), \phi, \psi, \eta \in L^{1}[0,1]$ are nonnegative and $f, g, h \in$ $C([0,1] \times[0, \infty) \times[0, \infty),[0, \infty))$ and $D$ is the standard Riemann-Liouville fractional derivative.

Also, we provide some examples to demonstrate the validity of our results.

Keywords: Tripled system, fractional differential equation, integral boundary conditions, existence and nonexistence of positive solutions.

2010 MSC: 34A08, 34B37, 35R11

Email addresses: hojat.afshari@yahoo.com (Hojjat Afshari), hadishojaat@yahoo.com (Hadi Shojaat), mansorehmoradi@gmail.com (Mansoureh Siahkali Moradi) 


\section{Introduction}

E. Karapınar and coauthors obtained some fixed point results and applied them to proving the existence and uniqueness of positive solutions for functional boundary value problem (see [1]-[17], [15], [26]). In recent years, some systems of nonlinear fractional differential equations were examined by many authors, [18]-25] and other references. In [27], Su investigated some conditions for the existence of solutions for a coupled system of two-point fractional boundary value problem.

In 31 the authors studied the existence and nonexistence of positive solutions to boundary values problem for a coupled system of nonlinear fractional differential equations as follows:

$$
\left\{\begin{array}{lll}
D^{\alpha} u(\zeta)+a(\zeta) f(\zeta, v(\zeta))=0, & u(0)=0, & u(1)=\int_{0}^{1} \phi(\zeta) u(\zeta) d \zeta \\
D^{\beta} v(\zeta)+b(\zeta) g(\zeta, u(\zeta))=0, & v(0)=0, & v(1)=\int_{0}^{1} \psi(\zeta) v(\zeta) d \zeta
\end{array}\right.
$$

where $0 \leq \zeta \leq 1,1<\alpha, \beta \leq 2, a, b \in C((0,1),[0, \infty)), \phi, \psi \in L^{1}[0,1]$ are nonnegative and $f, g \in$ $C([0,1] \times[0, \infty),[0, \infty))$ and $D$ is the standard Riemann-Liouville fractional derivative.

In this paper we study the equations

$$
\left\{\begin{array}{lll}
D^{\alpha} u(\zeta)+a(\zeta) f(\zeta, v(\zeta), \omega(\zeta))=0, & u(0)=0, & u(1)=\int_{0}^{1} \phi(\zeta) u(\zeta) d \zeta \\
D^{\beta} v(\zeta)+b(\zeta) g(\zeta, u(\zeta), \omega(\zeta))=0, & v(0)=0, & v(1)=\int_{0}^{1} \psi(\zeta) v(\zeta) d \zeta \\
D^{\gamma} \omega(\zeta)+c(\zeta) h(\zeta, u(\zeta), v(\zeta))=0, & \omega(0)=0, & \omega(1)=\int_{0}^{1} \eta(\zeta) \omega(\zeta) d \zeta
\end{array}\right.
$$

where $0 \leq \zeta \leq 1,1<\alpha, \beta, \gamma \leq 2, a, b, c \in C((0,1),[0, \infty)), \phi, \psi, \eta \in L^{1}[0,1]$ are nonnegative and $f, g, h \in C([0,1] \times[0, \infty) \times[0, \infty),[0, \infty))$ and $D$ is the standard Riemann-Liouville fractional derivative.

Definition 1.1. [28, 29] The Riemann-Liouville fractional derivative for a continuous function $f$ is defined by

$$
D^{\nu} f(\tau)=\frac{1}{\Gamma(n-\nu)}\left(\frac{d}{d \tau}\right)^{n} \int_{0}^{\tau} \frac{f(\zeta)}{(\tau-\zeta)^{\nu-n+1}} d \zeta, \quad(n=[\nu]+1)
$$

where the right-hand side is point-wise defined on $(0, \infty)$.

Definition 1.2. [28, 29] Let $[a, b]$ be an interval in $\mathbb{R}$ and $\nu>0$. The Riemann-Liouville fractional order integral of a function $f \in L^{1}([a, b], \mathbb{R})$ is defined by

$$
I_{a}^{\nu} f(\tau)=\frac{1}{\Gamma(\nu)} \int_{a}^{\tau} \frac{f(\zeta)}{(\tau-\zeta)^{1-\nu}} d \zeta
$$

whenever the integral exists.

Lemma 1.3. (Nonlinear Differentiation of Leray-Schauder Type, [32]). Let $E$ be a Banach space with $C \subset E$ closed and convex. Let $U$ be a relatively open subset of $C$ with $0 \in U$ and let $T: U \rightarrow C$ be a continuous and compact mapping. Then either

(a) the mapping $T$ has a fixed point in $U$, or (b) there exist $u \in \partial U$ and $\lambda \in(0,1)$ with $u=\lambda T u$.

Lemma 1.4. (Fixed-Point Theorem of Cone Expansion and Compression of Norm Type, See [33]). Let P be a cone of real Banach space $E$, and let $\Omega_{1}$ and $\Omega_{2}$ be two bounded open sets in $E$ such that $0 \in \Omega_{1} \subset \overline{\Omega_{1}} \subset \Omega_{2}$. Let operator $A: P \cap\left(\overline{\Omega_{2}}-\Omega_{1}\right) \rightarrow P$ be completely continuous operator. Suppose that one of the two conditions holds: 
$\left(i_{1}\right)\|A u\| \leq\|u\|$, for all $u \in P \cap \partial \Omega_{1} ;\|A u\| \geq\|u\|$, for all $u \in P \cap \partial \Omega_{2} ;$

$\left(i_{2}\right)\|A u\| \geq\|u\|$, for all $u \in P \cap \partial \Omega_{1} ;\|A u\| \leq\|u\|$, for all $u \in P \cap \partial \Omega_{2}$.

Then $A$ has at least one fixed point in $P \cap\left(\overline{\Omega_{2}}-\Omega_{1}\right)$.

Lemma 1.5. Assume that $\int_{0}^{1} \zeta^{\nu-1} \phi(\zeta) d \zeta \neq 1$. Then for any $\sigma \in C[0,1]$, the unique solution of boundary value problem

$$
\left\{\begin{array}{l}
D^{\nu} u(\zeta)+\sigma(\zeta)=0, \quad 0<\tau<1 \\
u(0)=0, \quad u(1)=\int_{0}^{1} \phi(\zeta) u(\zeta) d \zeta
\end{array}\right.
$$

is given by

$$
u(\zeta)=\int_{0}^{1} G_{1 \nu}(\zeta, \tau) \sigma(\tau) d \tau
$$

where

$$
G_{1 \nu}(\zeta, \tau)=G_{2 \nu}(\zeta, \tau)+G_{3 \nu}(\zeta, \tau), \quad(\zeta, \tau) \in[0,1] \times[0,1]
$$

with

$$
G_{2 \nu}(\zeta, \tau)=\frac{1}{\Gamma(\nu)}\left\{\begin{array}{l}
\zeta^{\nu-1}(1-\tau)^{\nu-1}-(\zeta-\tau)^{\nu-1}, 0 \leq \tau \leq \zeta \leq 1 \\
\zeta^{\nu-1}(1-\tau)^{\nu-1}, \quad 0 \leq \zeta \leq \tau \leq 1
\end{array}\right.
$$

and

$$
G_{3 \nu}(\zeta, \tau)=\frac{\zeta^{\nu-1}}{1-\int_{0}^{1} \phi(\zeta) \zeta^{\nu-1} d \zeta} \int_{0}^{1} G_{2 \nu}(\zeta, \tau) \phi(\zeta) d \zeta .
$$

We call $G=\left(G_{1 \nu}, G_{1 \nu^{\prime}}, G_{1 \nu^{\prime \prime}}\right)$ the Green's functions of the boundary value problem (2).

Lemma 1.6. If $\int_{1}^{0} \varphi(\tau) \tau^{\nu-1} d \tau \in[0,1)$, the function $G_{1 \nu}(\tau, \zeta)$ defined by 3 satisfies

$\left(i_{1}\right) G_{1 \nu}(\tau, \zeta) \geq 0$ is continuous for all $\tau, \zeta \in[0,1], G_{1 \nu}(\tau, \zeta)>0$ for all $\tau, \zeta \in(0,1)$;

$\left(i_{2}\right) G_{1 \nu}(\tau, \zeta) \leq G_{1 \nu}(\zeta)$ for each $\tau, \zeta \in(0,1)$, and $\min _{\tau \in[\theta, 1-\theta]} G_{1 \nu}(\tau, \zeta) \geq G_{1 \nu}(\zeta)$, where $\theta \in\left(0, \frac{1}{2}\right)$ and

$$
G_{1 \nu}(\zeta)=G_{2 \nu}(\zeta, \zeta)+G_{3 \nu}(1, \zeta), \quad \Upsilon_{\nu}=\theta^{\nu-1}
$$

We will discuss the existence of positive solutions for boundary value problem (2). First of all, we define the Banach space

$$
\begin{array}{ll}
X & =\{u(\zeta) \mid u(\zeta) \in C[0,1]\} \quad \text { endowed with the norm }\|u\|_{X}=\max _{\zeta \in[0,1]}|u|, \\
Y=\{v(\zeta) \mid v(\zeta) \in C[0,1]\} & \text { endowed with the norm }\|v\|_{Y}=\max _{\zeta \in[0,1]}|v|, \\
Z=\{\omega(\zeta) \mid \omega(\zeta) \in C[0,1]\} & \text { endowed with the norm }\|\omega\|_{Z}=\max _{\zeta \in[0,1]}|\omega| .
\end{array}
$$

For $(u, v, \omega) \in X \times Y \times Z$, let $\|(u, v, \omega)\|_{X \times Y \times Z}=\max \left\{\|u\|_{X},\|v\|_{Y},\|\omega\|_{Z}\right\}$. Clearly, $\left(X \times Y \times Z,\|(u, v, \omega)\|_{X \times Y \times Z}\right)$ is a Banach space. Define, $P=\{(u, v, \omega) \in X \times Y \times Z \mid u(\zeta) \geq 0, v(\zeta) \geq 0, \omega(\zeta) \geq 0\}$, then the cone $P \subset X \times Y \times Z$. Let $J_{\theta}=[\theta, 1-\theta]$ for $\theta \in\left(0, \frac{1}{2}\right)$ and

$$
\begin{aligned}
& K=\left\{\begin{array}{l}
(u, v, \omega) \in P, \min _{\tau \in J_{\theta}} u(\tau) \geq \Upsilon_{\alpha}\|u\|, \\
\min _{\tau \in J_{\theta}} v(\tau) \geq \Upsilon_{\beta}\|v\|, \min _{\tau \in J_{\theta}} \omega(\tau) \geq \Upsilon_{\gamma}\|\omega\|
\end{array}\right\}, \\
& K_{r}=\{(u, v, \omega) \in K,\|(u, v, \omega)\| \leq r\}, \\
& \partial K_{r}=\{(u, v, \omega) \in K,\|(u, v, \omega)\|=r\} .
\end{aligned}
$$

From Lemma 1.5, we can obtain the following lemma. 
Lemma 1.7. Suppose that $f(t, v, \omega), g(t, u, \omega)$ and $h(t, u, v)$ are continuous, then $(u, v, \omega) \in X \times Y \times Z$ is a solution of B.V.P (2) if and only if $(u, v, \omega) \in X \times Y \times Z$ is a solution of the integral equations

$$
\left\{\begin{array}{l}
u(\zeta)=\int_{0}^{1} G_{1 \alpha}(\zeta, \tau) a(\tau) f(\tau, v(\tau), \omega(\tau)) d \tau \\
v(\zeta)=\int_{0}^{1} G_{1 \beta}(\zeta, \tau) b(\tau) g(\tau, u(\tau), \omega(\tau)) d \tau \\
\omega(\zeta)=\int_{0}^{1} G_{1 \gamma}(\zeta, \tau) c(\tau) h(\tau, u(\tau), v(\tau)) d \tau
\end{array}\right.
$$

Let $T: X \times Y \times Z \rightarrow X \times Y \times Z$ be the operator defined as

$$
\begin{aligned}
& T(u, v, \omega)=\left(\int_{0}^{1} G_{1 \alpha}(\zeta, \tau) a(\tau) f(\tau, v(\tau), \omega(\tau)) d \tau\right. \\
& \left.\int_{0}^{1} G_{1 \beta}(\zeta, \tau) b(\tau) g(\tau, u(\tau), \omega(\tau)) d \tau, \int_{0}^{1} G_{1 \gamma}(\zeta, \tau) c(\tau) h(\tau, u(\tau), v(\tau)) d \tau\right) \\
& =:\left(T_{1} u(\zeta), T_{2} v(\zeta), T_{3} \omega(\zeta)\right),
\end{aligned}
$$

then by Lemma (1.7), the fixed point of operator T coincides with the solution of system (2).

Lemma 1.8. Let $f(\tau, v, v), g(\tau, u, u)$ and $h(\tau, \omega, \omega)$ be continuous on $[0,1] \times[0,+\infty) \times[0,+\infty) \rightarrow[0,+\infty)$, then $T: P \rightarrow P, T: K \rightarrow K$ defined by (4) are completely continuous.

Proof. Since Lemma (1.8) is similar to Lemma (1.8) in [2] and [30] we omit the proof Lemma (1.8).

Theorem 1.9. Assume that $a(\tau), b(\tau)$ and $c(\tau)$, are continuous on $(0,1) \rightarrow[0,+\infty)$ and $f(\tau, v(\tau), \omega(\tau)), g(\tau, u(\tau), \omega(\tau))$ and $h(\tau, u(\tau), v(\tau))$ are continuous on $[0,1] \times[0, \infty) \times$ $[0, \infty) \rightarrow[0, \infty)$, and there exist three positive functions $m(\tau), n(\tau)$ and $k(\tau)$ that satisfy

$\left(L_{1}\right) f\left(\tau, v_{2}, \omega_{2}\right)-f\left(\tau, v_{1}, \omega_{1}\right) \leq m(\tau) \max \left\{\left|v_{2}-v_{1}\right|,\left|\omega_{2}-\omega_{1}\right|\right\}$,

$\left(L_{2}\right) g\left(\tau, u_{2}, \omega_{2}\right)-g\left(\tau, u_{1}, \omega_{1}\right) \leq k(\tau) \max \left\{\left|u_{2}-u_{1}\right|,\left|\omega_{2}-\omega_{1}\right|\right\}$,

$\left(L_{3}\right) h\left(\tau, u_{2}, v_{2}\right)-h\left(\tau, u_{1}, v_{1}\right) \leq n(\tau) \max \left\{\left|v_{2}-v_{1}\right|,\left|u_{2}-u_{1}\right|\right\}$,

for $\tau \in(0,1), v_{1}, v_{2}, \omega_{1}, \omega_{2}, u_{1}, u_{2} \in(0,+\infty)$.

Then system (2) has a unique positive solution if

$$
\begin{aligned}
& \rho=\int_{0}^{1} G_{1 \alpha}(\tau) a(\tau) m(\tau) d \tau<1, \\
& \theta=\int_{0}^{1} G_{1 \beta}(\tau) b(\tau) k(\tau) d \tau<1 \\
& \kappa=\int_{0}^{1} G_{1 \gamma}(\tau) c(\tau) n(\tau) d \tau<1 .
\end{aligned}
$$

Proof. For all $(u, v, \omega) \in P$ by the nonnegativeness of $G(\zeta, \tau)$ and $a(\tau), b(\tau), c(\tau), f(\tau, v(\tau), \omega(\tau)), g(\tau, u(\tau), \omega(\tau))$, $h(\tau, u(\tau), v(\tau))$, we have $T(u, v, \omega) \geq 0$. Hence, $T(P) \subset P$. From Lemma 1.6, we obtain

$$
\begin{aligned}
& \left\|T_{1} v_{2}-T_{1} v_{1}\right\|=\max _{\zeta \in[0,1]}\left|T_{1} v_{2}-T_{1} v_{1}\right| \\
& =\max _{\zeta \in[0,1]}\left|\left(\int_{0}^{1} G_{1 \alpha}(\zeta, \tau) a(\tau)\left[f\left(\tau, v_{2}(\tau), \omega(\tau)\right)-f\left(\tau, v_{1}(\tau), \omega(\tau)\right)\right]\right) d \tau\right| \\
& \leq\left(\int_{0}^{1} G_{1 \alpha}(\tau) a(\tau) m(\tau)\right) d \tau \mid\left\|v_{2}-v_{1}\right\|=\rho\left\|v_{2}-v_{1}\right\|
\end{aligned}
$$


Similarly,

$$
\left\|T_{2} u_{2}-T_{2} u_{1}\right\| \leq \theta\left\|u_{2}-u_{1}\right\|
$$

and

$$
\left\|T_{3} \omega_{2}-T_{3} \omega_{1}\right\| \leq \kappa\left\|\omega_{2}-\omega_{1}\right\|
$$

From (7), (8) to (9), we get

$$
\left\|T\left(u_{2}, v_{2}, \omega_{2}\right)-T\left(u_{1}, v_{1}, \omega_{1}\right)\right\| \leq \max (\rho, \theta, \kappa)\left\|\left(u_{2}, v_{2}, \omega_{2}\right)-\left(u_{1}, v_{1}, \omega_{1}\right)\right\|
$$

From Lemma (1.8), $T$ is completely continuous, by Banach fixed point theorem, the operator $T$ has a unique fixed point in $P$, which is the unique positive solution of system (2). This completes the proof.

Theorem 1.10. Assume that $a(\tau), b(\tau)$ and $c(\tau)$, are continuous on

$(0,1) \rightarrow[0,+\infty)$ and $f(\tau, v(\tau), \omega(\tau)), g(\tau, u(\tau), \omega(\tau))$ and $h(\tau, u(\tau), v(\tau))$ are continuous on $[0,1] \times[0, \infty) \times$ $[0, \infty) \rightarrow[0, \infty)$, and satisfy

$\left(L_{4}\right)|f(\tau, v(\tau), \omega(\tau))| \leq a_{1}(\tau)+a_{2}(\tau) \max \{|v(\tau)|,|\omega(\tau)|\}$,

$\left(L_{5}\right)|g(\tau, u(\tau), \omega(\tau))| \leq b_{1}(\tau)+b_{2}(\tau) \max \{|u(\tau)|,|\omega(\tau)|\}$,

$\left(L_{6}\right)|h(\tau, u(\tau), v(\tau))| \leq c_{1}(\tau)+c_{2}(\tau) \max \{|v(\tau)|,|u(\tau)|\}$,

$\left(L_{7}\right) A_{1}=\int_{0}^{1} G_{1 \alpha}(\tau) a(\tau) a_{2}(\tau) d \tau<1, B_{1}=\int_{0}^{1} G_{1 \alpha}(\tau) a(\tau) a_{1}(\tau) d \tau<\infty$,

$\left(L_{8}\right) A_{2}=\int_{0}^{1} G_{1 \beta}(\tau) b(\tau) b_{2}(\tau) d \tau<1, B_{2}=\int_{0}^{1} G_{1 \beta}(\tau) b(\tau) b_{1}(\tau) d \tau<\infty$,

(L) $A_{3}=\int_{0}^{1} G_{1 \gamma}(\tau) c(\tau) c_{2}(\tau) d \tau<1, B_{3}=\int_{0}^{1} G_{1 \gamma}(\tau) c(\tau) c_{1}(\tau) d \tau<\infty$.

Then the system (2) has at least one positive solution $(u, v, \omega)$ in

$$
Q=\left\{(u, v, \omega) \in P:\|(u, v, \omega)\|<\min \left(\frac{A_{1}}{1-B_{1}}, \frac{A_{2}}{1-B_{2}}, \frac{A_{3}}{1-B_{3}}\right)\right\} .
$$

Proof. Let $Q=\{(u, v, \omega) \in P:\|(u, v, \omega)\|<r\}$ with

$$
r=\min \left(\frac{A_{1}}{1-B_{1}}, \frac{A_{2}}{1-B_{2}}, \frac{A_{3}}{1-B_{3}}\right) .
$$

Define the operator $T: Q \rightarrow P$ as (4). Let $(u, v, \omega) \in Q$, that is,

$\|(u, v, \omega)\|<r$. Then

$$
\begin{aligned}
\left\|T_{1} u\right\| & =\max _{\zeta \in[0,1]}\left|\int_{0}^{1} G_{1 \alpha}(\zeta, \tau) a(\tau) f(\tau, v(\tau), \omega(\tau)) d \tau\right| \\
& \leq \int_{0}^{1} G_{1 \alpha}(\tau) a(\tau)\left(a_{1}(\tau)+a_{2}(\tau)|v(\tau)|\right) d \tau \\
& \leq \int_{0}^{1} G_{1 \alpha}(\tau) a(\tau) a_{1}(\tau) d \tau+\int_{0}^{1} G_{1 \alpha}(\tau) a(\tau) a_{2}(\tau) d \tau\|v(\tau)\| \\
& =B_{1}+A_{1}\|v(\tau)\| \leq r .
\end{aligned}
$$

Similarly, $\left\|T_{2} v\right\| \leq r,\left\|T_{3} \omega\right\| \leq r$. So, $T(u, v, \omega) \leq(r, r, r)$ and hence $T(u, v, \omega) \in \bar{Q}$. From Lemma (1.8), we have $T: Q \rightarrow \bar{Q}$ is completely continuous. Consider the eigenvalue problem

$$
(u, v, \omega)=\lambda T(u, v, \omega), \quad \lambda \in(0,1) .
$$


Under the assumption that $(u, v, \omega)$ is a solution of 10$)$ for $\lambda \in(0,1)$, we have

$$
\begin{aligned}
\|u\|=\left\|\lambda T_{1} u\right\| & =\max _{\zeta \in[0,1]}\left|\int_{0}^{1} G_{1 \alpha}(\zeta, \tau) a(\tau) f(\tau, v(\tau), \omega(\tau)) d \tau\right| \\
& \leq \int_{0}^{1} G_{1 \alpha}(\tau) a(\tau)\left(a_{1}(\tau)+a_{2}(\tau)|v(\tau)|\right) d \tau \\
& \leq \int_{0}^{1} G_{1 \alpha}(\tau) a(\tau) a_{1}(\tau) d \tau+\int_{0}^{1} G_{1 \alpha}(\tau) a(\tau) a_{2}(\tau) d \tau\|v(\tau)\| \\
& =B_{1}+A_{1}\|v(\tau)\| \leq r .
\end{aligned}
$$

Similarly, $\|v\|=\left\|T_{2} \lambda v\right\| \leq r,\|\omega\|=\left\|T_{3} \lambda \omega\right\| \leq r$, so, $\|(u, v, \omega)\| \leq r$, which shows that $(u, v, \omega) \in \partial Q$. By Lemma 1.3, $T$ has a fixed point in $Q$. We complete the proof of theorem 1.10 .

Remark 1.11. In the following we need the following assumptions and some notations:

$\left(B_{1}\right) a, b, c \in C((0,1),[0, \infty)), a(\tau) \neq 0, b(\tau) \neq 0, c(\tau) \neq 0$ on any subinterval of $(0,1)$ and

$$
0<\int_{0}^{1} G_{1 \alpha}(\tau) a(\tau) d \tau<\infty
$$

$0<\int_{0}^{1} G_{1 \beta}(\tau) b(\tau) d \tau<\infty$ and $0<\int_{0}^{1} G_{1 \gamma}(\tau) c(\tau) d \tau<\infty$ where $G_{1 \alpha}, G_{1 \beta}$ and $G_{1 \gamma}$ are defined in Lemma 1.6;

$\left(B_{2}\right) f, g, h \in C([0,1] \times[0, \infty) \times[0, \infty),[0, \infty))$ and $f(\zeta, 0,0)=0$,

$g(\zeta, 0,0)=0$ and $h(\zeta, 0,0)=0$ uniformly with respect to $\zeta$ on $[0,1]$

(B) $\lambda, \mu, \nu \in[0,1)$ where $\lambda, \mu, \nu$ is defined as follows:

$$
\lambda=\int_{0}^{1} \phi(\zeta) \zeta^{\alpha-1} d \zeta, \quad \mu=\int_{0}^{1} \psi(\zeta) \zeta^{\beta-1} d \zeta \quad \text { and } \quad \nu=\int_{0}^{1} \varphi(\zeta) \zeta^{\gamma-1} d \zeta
$$

let

$$
f^{\delta}=\limsup _{u \rightarrow \delta} \max _{\zeta \in[0,1]} \frac{f(\zeta, u, u)}{u}, \quad f_{\delta}=\liminf _{u \rightarrow \delta} \min _{\zeta \in[0,1]} \frac{f(\zeta, u, u)}{u}
$$

where $\delta$ denotes 0 or $\infty$, and

$$
\sigma_{1}=\int_{0}^{1} G_{1 \alpha}(\tau) a(\tau) d \tau, \quad \sigma_{2}=\int_{0}^{1} G_{1 \beta}(\tau) b(\tau) d \tau \text { and } \sigma_{3}=\int_{0}^{1} G_{1 \gamma}(\tau) c(\tau) d \tau .
$$

Theorem 1.12. Assume that $\left(B_{1}\right)-\left(B_{3}\right)$ hold. And supposes that one of the following conditions is satisfied: $\left(H_{1}\right) f_{0}>\frac{1}{\Upsilon_{\alpha}^{2} \int_{\theta}^{1-\theta} G_{1 \alpha}(\tau) a(\tau) d \tau}$ and $f^{\infty}<\frac{1}{\sigma_{1}}$ (particularly, $f^{0}=\infty$ and $f^{\infty}=0$ );

$g_{0}>\frac{1}{\Upsilon_{\beta}^{2} \int_{\theta}^{1-\theta} G_{1 \beta}(\tau) b(\tau) d \tau}$ and $g^{\infty}<\frac{1}{\sigma_{2}}$ (particularly, $g^{0}=\infty$ and $g^{\infty}=0$ );

$h_{0}>\frac{1}{\Upsilon_{\gamma}^{2} \int_{\theta}^{1-\theta} G_{1 \gamma}(\tau) c(\tau) d \tau}$ and $h^{\infty}<\frac{1}{\sigma_{3}}$ (particularly, $h^{0}=\infty$ and $h^{\infty}=0$ ).

$\left(H_{2}\right)$ There exist two constants $r_{2}, R_{2}$ with $0<r_{2} \leq R_{2}$ such that $f(\zeta, .,),. g(\zeta, .,$.$) and h(\zeta, .,$.$) are nonde-$ creasing on $\left[0, R_{2}\right]$ for all $\zeta \in[0,1]$,

$$
\begin{gathered}
f\left(\zeta, \Upsilon_{\alpha} r_{2}, \Upsilon_{\alpha} r_{2}\right) \geq \frac{r_{2}}{\Upsilon_{\alpha}^{2} \int_{\theta}^{1-\theta} G_{1 \alpha}(\tau) a(\tau) d \tau}, \\
g\left(\zeta, \Upsilon_{\beta} r_{2}, \Upsilon_{\beta} r_{2}\right) \geq \frac{r_{2}}{\Upsilon_{\beta}^{2} \int_{\theta}^{1-\theta} G_{1 \beta}(\tau) b(\tau) d \tau}, \\
h\left(\zeta, \Upsilon_{\gamma} r_{2}, \Upsilon_{\gamma} r_{2}\right) \geq \frac{r_{2}}{\Upsilon_{\gamma}^{2} \int_{\theta}^{1-\theta} G_{1 \gamma}(\tau) c(\tau) d \tau}
\end{gathered}
$$

and $f\left(\zeta, R_{2}, R_{2}\right) \leq \frac{R_{2}}{\sigma_{1}}, g\left(\zeta, R_{2}, R_{2}\right) \leq \frac{R_{2}}{\sigma_{2}}, h\left(\zeta, R_{2}, R_{2}\right) \leq \frac{R_{2}}{\sigma_{3}}$ for all $\zeta \in[0,1]$. Then boundary value problem (2) has at least one positive solution. 
Proof. Let $T$ be cone preserving completely continuous that is defined by (4).

Case1. The condition $\left(H_{1}\right)$ holds. Considering $f_{0}>\frac{1}{\Upsilon_{\alpha}^{2} \int_{\theta}^{1-\theta} G_{1 \alpha}(\tau) a(\tau) d \tau}$, there exists $r_{1}>0$ such that $f(t, v, v)=\left(f_{0}-\varepsilon_{1}\right) v$, for all $t \in[0,1], v \in\left[0, r_{1}\right]$, where $\varepsilon_{1}>0$, satisfies

$$
\left(f_{0}-\varepsilon_{1}\right) \Upsilon_{\alpha}^{2} \int_{\theta}^{1-\theta} G_{1 \alpha}(\tau) a(\tau) d \tau \geq 1
$$

Then, for $t \in[0,1],(u, v, \omega) \in \partial K_{r_{1}}$, we get

$$
\begin{aligned}
T_{1} v(t) & =\int_{0}^{1} G_{1 \alpha}(\zeta, \tau) a(\tau) f(\tau, v(\tau), v(\tau)) d \tau \\
& \geq \Upsilon_{\alpha} \int_{0}^{1} G_{1 \alpha}(\tau) a(\tau) f(\tau, v(\tau), v(\tau)) d \tau \\
& \geq \Upsilon_{\alpha} \int_{0}^{1} G_{1 \alpha}(\tau) a(\tau)\left(f_{0}-\varepsilon_{1}\right) v(\tau) d \tau \\
& \geq\left(f_{0}-\varepsilon_{1}\right) \Upsilon_{\alpha}^{2} \int_{0}^{1} G_{1 \alpha}(\tau) a(\tau) d \tau\|v\| \\
& \geq\|v\|
\end{aligned}
$$

Similarly, we have $T_{2} \omega(t) \geq\|\omega\|, T_{3} u(t) \geq\|u\|$ that is $(u, v, \omega) \in \partial K_{r_{1}}$ implies that

$$
\|T(u, v, \omega)\| \geq\|(u, v, \omega)\| .
$$

On the other hand, for $f^{\infty}<1 / \sigma_{1}$, there exists $\bar{R}_{1}>0$ such that $f(t, v, v)=\left(f_{\infty}+\varepsilon_{2}\right) v$, for $t \in[0,1]$, $v \in\left(R_{1},+\infty\right)$, where $\varepsilon_{2}>0$ satisfies $\sigma_{1}\left(f^{\infty}+\varepsilon_{2}\right)=1$. Set $M=\max _{t \in[0,1], v \in\left[0, R_{1}\right]} f(t, v, v)$, then $f(t, v, v)=M+\left(f^{\infty}+\varepsilon_{2}\right) v$. Choose

$R_{1}>\max \left\{r_{1}, \bar{R}_{1}, M \sigma_{1}\left(1-\sigma_{1}\left(f^{\infty}+\varepsilon_{2}\right)\right)^{-1}\right\}$. Then, for $t \in[0,1],(u, v, \omega) \in \partial K_{R_{1}}$, we get

$$
\begin{aligned}
T_{1} v(t) & =\int_{0}^{1} G_{1 \alpha}(\zeta, \tau) a(\tau) f(\tau, v(\tau), v(\tau)) d \tau \\
& \leq \int_{0}^{1} G_{1 \alpha}(\tau) a(\tau)\left(M+\left(f^{\infty}+\varepsilon_{2}\right)\right) v(\tau) d \tau \\
& \leq M \int_{0}^{1} G_{1 \alpha}(\tau) a(\tau) d \tau+\int_{0}^{1} G_{1 \alpha}(\tau) a(\tau)\left(f^{\infty}+\varepsilon_{2}\right) d \tau\|v\| \\
& \leq R_{1}-\sigma_{1}\left(f^{\infty}+\varepsilon_{2}\right) R_{1}+\left(f^{\infty}+\varepsilon_{2}\right) \sigma_{1}\|v\| \\
& \leq R_{1} .
\end{aligned}
$$

Similarly, we have $T_{3} u(t) \leq\|u\|, T_{2} \omega(t) \leq\|\omega\|$ that is $(u, v, \omega) \in \partial K_{R_{1}}$ implies that

$$
\|T(u, v, \omega)\| \leq\|(u, v, \omega)\| .
$$

Case2. The condition $\left(H_{2}\right)$ holds. For $(u, v, \omega) \in K$, from the definition of $K$, we obtain that

$$
\min _{t \in J_{\theta}} u(t) \geq \Upsilon_{\alpha}\|u\|, \min _{t \in J_{\theta}} v(t) \geq \Upsilon_{\beta}\|v\|, \min _{t \in J_{\theta}} \omega(t) \geq \Upsilon_{\gamma}\|\omega\| .
$$


Therefore, for $(u, v, \omega) \in \partial K_{r_{2}}$, we have $\|(u, v, \omega)\|=r_{2}$ for $t \in J_{\theta}$. From $\left(H_{2}\right)$, we have

$$
\begin{aligned}
T_{1} v(t) & =\int_{0}^{1} G_{1 \alpha}(\zeta, \tau) a(\tau) f(\tau, v(\tau), v(\tau)) d \tau \\
& \geq \Upsilon_{\alpha} \int_{\theta}^{1-\theta} G_{1 \alpha}(\tau) a(\tau) f(\tau, v(\tau), v(\tau)) d \tau \\
& \geq \Upsilon_{\alpha} \frac{r_{2}}{\Upsilon_{\alpha} \int_{\theta}^{1-\theta} G_{1 \alpha}(\tau) a(\tau) d \tau} \int_{\theta}^{1-\theta} G_{1 \alpha}(\tau) a(\tau) d \tau \\
& =r_{2} .
\end{aligned}
$$

Similarly, we have $T_{3} u(t) \geq r_{2}, T_{2} \omega(t) \geq r_{2}$ that is $(u, v, \omega) \in \partial K_{r_{2}}$ implies that

$$
\|T(u, v, \omega)\| \geq\|(u, v, \omega)\|
$$

On the other hand, for $(u, v, \omega) \in \partial K_{R_{2}}$, we have that $(u, v, \omega)=R_{2}$ for $t \in[0,1]$, from $\left(H_{2}\right)$, we obtain

$$
\begin{aligned}
T_{1} v(t) & =\int_{0}^{1} G_{1 \alpha}(\zeta, \tau) a(\tau) f(\tau, v(\tau), v(\tau)) d \tau \\
& \leq \Upsilon_{\alpha} \int_{0}^{1} G_{1 \alpha}(\tau) a(\tau) f(\tau, v(\tau), v(\tau)) d \tau \\
& \leq \frac{R_{2}}{\sigma_{1}} \Upsilon_{\alpha} \int_{0}^{1} G_{1 \alpha}(\tau) a(\tau) d \tau \\
& =R_{2}
\end{aligned}
$$

Similarly, we have $T_{3} u(t) \leq R_{2}, T_{2} \omega(t) \leq R_{2}$ that is $(u, v, \omega) \in \partial K_{R_{2}}$ implies that

$$
\|T(u, v, \omega)\| \leq\|(u, v, \omega)\| .
$$

Applying Lemma 1.4 to 11 and $\left(12\right.$, or 13 and $\sqrt{14}$, yields that $T$ has a fixed point $(\bar{u}, \bar{v}, \bar{\omega}) \in \bar{K}_{r, R}$ or $(\bar{u}, \bar{v}, \bar{\omega}) \in \bar{K}_{r_{i}, R_{i}}(i=1,2)$ with $\bar{u}(t)=\Upsilon_{\alpha}\|u\|>0, \bar{v}(t)=\Upsilon_{\beta}\|\bar{v}\|>0$ and $\bar{\omega}(t)=\Upsilon_{\gamma}\|\bar{\omega}\|>0$. Thus it follows that boundary value problems (1.1) has a positive solution $(\bar{u}, \bar{v}, \bar{\omega})$. We complete the proof of Theorem 1.12,

Similarly, we have the following result.

Theorem 1.13. Assume that $\left(B_{1}\right)-\left(B_{3}\right)$ hold. And supposes that the following three conditions are satisfied:

$\left(H_{3}\right) f^{0}<\frac{1}{\sigma_{1}}$ and $f_{\infty}>\frac{1}{\Upsilon_{\alpha}^{2} \int_{\theta}^{1-\theta} G_{1 \alpha}(\tau) a(\tau) d \tau}$ (particularly, $f^{0}=0$ and $\left.f_{\infty}=\infty\right)$;

$g^{0}<\frac{1}{\sigma_{2}}$ and $g_{\infty}>\frac{1}{\Upsilon_{\beta}^{2} \int_{\theta}^{1-\theta} G_{1 \beta}(\tau) b(\tau) d \tau}$ (particularly, $g^{0}=0$ and $g_{\infty}=\infty$ );

$h^{0}<\frac{1}{\sigma_{3}}$ and $h_{\infty}>\frac{1}{\Upsilon_{\gamma}^{2} \int_{\theta}^{1-\theta} G_{1 \gamma}(\tau) c(\tau) d \tau}$ (particularly, $h^{0}=0$ and $\left.h_{\infty}=\infty\right)$.

Then boundary value problem (2) has at least one positive solution.

Theorem 1.14. Assume that $\left(B_{1}\right)-\left(B_{3}\right)$ hold. And supposes that the following two conditions are satisfied: 
$\left(H_{4}\right) f_{0}>\frac{1}{\Upsilon_{\alpha}^{2} \int_{\theta}^{1-\theta} G_{1 \alpha}(\tau) a(\tau) d \tau}$ and $f_{\infty}>\frac{1}{\Upsilon_{\alpha}^{2} \int_{\theta}^{1-\theta} G_{1 \alpha}(\tau) a(\tau) d \tau}$

(particularly, $f^{0}=f_{\infty}=\infty$ );

$g_{0}>\frac{1}{\Upsilon_{\beta}^{2} \int_{\theta}^{1-\theta} G_{1 \beta}(\tau) b(\tau) d \tau}$ and $g_{\infty}>\frac{1}{\Upsilon_{\beta}^{2} \int_{\theta}^{1-\theta} G_{1 \beta}(\tau) b(\tau) d \tau}$

(particularly, $g^{0}=g_{\infty}=\infty$ );

$h_{0}>\frac{1}{\Upsilon_{\gamma}^{2} \int_{\theta}^{1-\theta} G_{1 \gamma}(\tau) c(\tau) d \tau}$ and $h_{\infty}>\frac{1}{\Upsilon_{\gamma}^{2} \int_{\theta}^{1-\theta} G_{1 \gamma}(\tau) c(\tau) d \tau}$

(particularly, $h^{0}=h_{\infty}=\infty$ ).

$\left(H_{5}\right)$ there exists $b>0$ such that

$$
\max _{\zeta \in[0,1],(u, v, \omega) \in \partial K_{b}} f(\zeta, u, u)<b / \sigma_{1}, \max _{\zeta \in[0,1],(u, v, \omega) \in \partial K_{b}} g(\zeta, v, v)<b / \sigma_{2}
$$

and

$$
\max _{\zeta \in[0,1],(u, v, \omega) \in \partial K_{b}} h(\zeta, \omega, \omega)<b / \sigma_{3} .
$$

Then boundary value problem (2) has at least two positive solutions $\left(u_{1}, v_{1}, \omega_{1}\right)$, $\left(u_{2}, v_{2}, \omega_{2}\right)$, which satisfy

$$
0<\left\|\left(u_{1}, v_{1}, \omega_{1}\right)\right\|<b<\left\|\left(u_{2}, v_{2}, \omega_{2}\right)\right\| .
$$

Proof. We consider condition $\left(H_{4}\right)$. Choose $r, R$ with $0<r<b<R$.

If $f_{0}>\frac{1}{\Upsilon_{\alpha}^{2} \int_{\theta}^{1-\theta} G_{1 \alpha}(\tau) a(\tau) d \tau}, g_{0}>\frac{1}{\Upsilon_{\beta}^{2} \int_{\theta}^{1-\theta} G_{1 \beta}(\tau) b(\tau) d \tau}$ and $h_{0}>\frac{1}{\Upsilon_{\gamma}^{2} \int_{\theta}^{1-\theta} G_{1 \gamma}(\tau) c(\tau) d \tau}$, then similar to the proof of (11), we have

$$
\|T(u, v, \omega)\| \geq\|(u, v, \omega)\|,
$$

for

$$
(u, v, \omega) \in \partial K_{r}
$$

If $f_{\infty}>\frac{1}{\Upsilon_{\alpha}^{2} \int_{\theta}^{1-\theta} G_{1 \alpha}(\tau) a(\tau) d \tau}, g_{\infty}>\frac{1}{\Upsilon_{\beta}^{2} \int_{\theta}^{1-\theta} G_{1 \beta}(\tau) b(\tau) d \tau}$ and $h_{\infty}>\frac{1}{\Upsilon_{\gamma}^{2} \int_{\theta}^{1-\theta} G_{1 \gamma}(\tau) c(\tau) d \tau}$, then similar to the proof of (3.6), we have

$$
\|T(u, v, \omega)\| \geq\|(u, v, \omega)\|, \quad \text { for } \quad(u, v, \omega) \in \partial K_{R}
$$

On the other hand, together with $\left(H_{5}\right),(u, v, \omega) \in \partial K_{b}$, we have

$$
\begin{aligned}
T_{1} v(\zeta) & =\int_{0}^{1} G_{1 \alpha}(\zeta, \tau) a(\tau) f(\tau, v(\tau), \omega(\tau)) d \tau \\
& \leq \int_{0}^{1} G_{1 \alpha}(\tau) a(\tau) f(\tau, v(\tau), \omega(\tau)) d \tau \\
& <\frac{b}{\sigma_{1}} \int_{0}^{1} G_{1 \alpha}(\tau) a(\tau) d \tau \\
& =b .
\end{aligned}
$$

Similarly, we have $T_{3} u(\zeta)<b, T_{2} \omega(\zeta)<b$, that is $(u, v, \omega) \in \partial K_{b}$ implies that

$$
\|T(u, v, \omega)\|<\|(u, v, \omega)\| .
$$


Applying Lemma 1.4 to $160-(18)$ yields that $T$ has a fixed point $\left(u_{1}, v_{1}, \omega_{1}\right) \in \overline{\partial K_{r, b}}$, and a fixed point $\left(u_{2}, v_{2}, \omega_{2}\right) \in \overline{\partial K_{b, R}}$. Thus it follows that boundary value problem (2) has at least two positive solutions $\left(u_{1}, v_{1}, \omega_{1}\right)$ and $\left(u_{2}, v_{2}, \omega_{2}\right)$. Noticing (18), we have $\left(u_{1}, v_{1}, \omega_{1}\right) \neq b$ and $\left(u_{2}, v_{2}, \omega_{2}\right) \neq b$. Therefore (15) holds, and the proof is complete.

Similarly, we have the following results.

Theorem 1.15. Assume that $\left(B_{1}\right)-\left(B_{3}\right)$ hold. And supposes that the following conditions is satisfied:

$\left(H_{6}\right) f^{0}<1 / \sigma_{1}$ and $f^{\infty}<1 / \sigma_{1} ; g^{0}<1 / \sigma_{2}$ and $g^{\infty}<1 / \sigma_{2} ; h^{0}<1 / \sigma_{3}$ and $h^{\infty}<1 / \sigma_{3}$.

$\left(H_{7}\right)$ there exists $B>0$ such that

$$
\begin{array}{r}
\max _{\zeta \in[0,1],(u, v, \omega) \in \partial K_{B}} f(\zeta, u, u)>\frac{B}{\Upsilon_{\alpha} \int_{\theta}^{1-\theta} G_{1 \alpha}(\tau) a(\tau) d \tau}, \\
\max _{\zeta \in[0,1],(u, v, \omega) \in \partial K_{B}} g(\zeta, v, v)>\frac{B}{\Upsilon_{\beta} \int_{\theta}^{1-\theta} G_{1 \beta}(\tau) b(\tau) d \tau}, \\
\max _{\zeta \in[0,1],(u, v, \omega) \in \partial K_{B}} h(\zeta, \omega, \omega)>\frac{B}{\Upsilon_{\gamma} \int_{\theta}^{1-\theta} G_{1 \gamma}(\tau) c(\tau) d \tau},
\end{array}
$$

Then boundary value problem (2) has at least two positive solutions $\left(u_{1}, v_{1}, \omega_{1}\right)$, $\left(u_{2}, v_{2}, \omega_{2}\right)$, which satisfy

$$
0<\left\|\left(u_{1}, v_{1}, \omega_{1}\right)\right\|<B<\left\|\left(u_{2}, v_{2}, \omega_{2}\right)\right\| .
$$

Theorem 1.16. Assume that $\left(B_{1}\right)-\left(B_{3}\right)$ hold. If there exist $3 l$ positive numbers $d_{k}, D_{k}, k=1,2, \cdots, l$ with

$$
\begin{gathered}
d_{1}<\Upsilon_{\alpha} D_{1}<D_{1}<d_{2}<\Upsilon_{\alpha} D_{2}<D_{2}<\cdots<d_{l}<\Upsilon_{\alpha} D_{l}<D_{l}, \\
d_{1}<\Upsilon_{\beta} D_{1}<D_{1}<d_{2}<\Upsilon_{\beta} D_{2}<D_{2}<\cdots<d_{l}<\Upsilon_{\beta} D_{l}<D_{l}
\end{gathered}
$$

and

$$
d_{1}<\Upsilon_{\gamma} D_{1}<D_{1}<d_{2}<\Upsilon_{\gamma} D_{2}<D_{2}<\cdots<d_{l}<\Upsilon_{\gamma} D_{l}<D_{l}
$$

such that

$\left(H_{8}\right)$

$$
f(\zeta, u, u)>\frac{d_{k}}{\Upsilon_{\alpha} \int_{0}^{1} G_{1 \alpha}(\tau) a(\tau) d \tau},
$$

for

$$
(\zeta, u, u) \in[0,1] \times\left[\Upsilon_{\alpha} d_{k}, d_{k}\right] \times\left[\Upsilon_{\alpha} d_{k}, d_{k}\right]
$$

and

$$
f(\zeta, u, u)=\sigma_{1}^{-1} D_{k}
$$

for

$$
(\zeta, u, u) \in[0,1] \times\left[\Upsilon_{\alpha} D_{k}, D_{k}\right] \times\left[\Upsilon_{\alpha} D_{k}, D_{k}\right], k=1,2, \cdots, l
$$

Also

$$
g(\zeta, v, v)>\frac{d_{k}}{\Upsilon_{\beta} \int_{0}^{1} G_{1 \beta}(\tau) b(\tau) d \tau},
$$


for

$$
(\zeta, v, v) \in[0,1] \times\left[\Upsilon_{\beta} d_{k}, d_{k}\right] \times\left[\Upsilon_{\beta} d_{k}, d_{k}\right]
$$

and

$$
g(\zeta, v, v)=\sigma_{1}^{-1} D_{k}
$$

for

$$
(\zeta, v, v) \in[0,1] \times\left[\Upsilon_{\beta} D_{k}, D_{k}\right] \times\left[\Upsilon_{\beta} D_{k}, D_{k}\right], k=1,2, \cdots, l .
$$

And also

$$
h(\zeta, \omega, \omega)>\frac{d_{k}}{\Upsilon_{\gamma} \int_{0}^{1} G_{1 \gamma}(\tau) c(\tau) d \tau}
$$

for

$$
(\zeta, \omega, \omega) \in[0,1] \times\left[\Upsilon_{\gamma} d_{k}, d_{k}\right] \times\left[\Upsilon_{\gamma} d_{k}, d_{k}\right]
$$

and

$$
h(\zeta, \omega, \omega)=\sigma_{1}^{-1} D_{k}
$$

for

$$
(\zeta, \omega, \omega) \in[0,1] \times\left[\Upsilon_{\gamma} D_{k}, D_{k}\right] \times\left[\Upsilon_{\gamma} D_{k}, D_{k}\right], k=1,2, \cdots, l .
$$

Then boundary value problem (2) has at least l positive solutions $\left(u_{k}, v_{k}, \omega_{k}\right)$ which satisfy

$$
d_{k}<\left\|\left(u_{k}, v_{k}, \omega_{k}\right)\right\|<D_{k}, \quad k=1,2, \cdots, l .
$$

Theorem 1.17. Assume that $\left(B_{1}\right)-\left(B_{3}\right)$ hold. If there exist $3 l$ positive numbers $d_{k}, D_{k}, k=1,2, \cdots, l$ with $d_{1}<D_{1}<d_{2}<D_{2}<\cdots<d_{l}<D_{l}$ such that $\left(H_{9}\right) f(\zeta, .,),. g(\zeta, .,$.$) and h(\zeta, .,$.$) are nondecreasing on \left[0, D_{l}\right]$ for all $t \in[0,1]$. $\left(H_{10}\right)$

$$
f\left(\zeta, \Upsilon_{\alpha} d_{k}, \Upsilon_{\alpha} d_{k}\right) \geq \frac{d_{k}}{\Upsilon_{\alpha} \int_{\theta}^{1-\theta} G_{1 \alpha}(\tau) a(\tau) d \tau}
$$

and

$$
f\left(\zeta, D_{k}, D_{k}\right) \leq \sigma_{1}^{-1} D_{k}, k=1,2, \cdots, l .
$$

Also

$$
g\left(\zeta, \Upsilon_{\beta} d_{k}, \Upsilon_{\beta} d_{k}\right) \geq \frac{d_{k}}{\Upsilon_{\beta} \int_{\theta}^{1-\theta} G_{1 \beta}(\tau) b(\tau) d \tau}
$$

and

$$
g\left(\zeta, D_{k}, D_{k}\right) \leq \sigma_{1}^{-1} D_{k}, k=1,2, \cdots, l .
$$

And also

$$
h\left(\zeta, \Upsilon_{\gamma} d_{k}, \Upsilon_{\gamma} d_{k}\right) \geq \frac{d_{k}}{\Upsilon_{\gamma} \int_{\theta}^{1-\theta} G_{1 \gamma}(\tau) c(\tau) d \tau}
$$

and

$$
h\left(\zeta, D_{k}, D_{k}\right) \leq \sigma_{1}^{-1} D_{k}, k=1,2, \cdots, l .
$$


Then boundary value problem (2) has at least l positive solutions $\left(u_{k}, v_{k}, \omega_{k}\right)$ which satisfy

$$
d_{k}<\left\|\left(u_{k}, v_{k}, \omega_{k}\right)\right\|<D_{k}, \quad k=1,2, \cdots, l .
$$

Now the nonexistence of positive solutions for boundary value problem (2).

Theorem 1.18. Suppose $\left(B_{1}\right)-\left(B_{3}\right)$ hold, $f(\zeta, u, u)<\sigma_{1}^{1} u, g(\zeta, v, v)<\sigma_{2}^{1} v$ and $h(\zeta, \omega, \omega)<\sigma_{3}^{1} \omega$ for all $\zeta \in[0,1], u>0, v>0$ and $\omega>0$ then boundary value problem (2) has no positive solution.

Proof. Assume to the contrary that $(u, v, \omega)$ is a positive solution of the boundary value problem (2). Then $(u, v, \omega) \in K, u>0, v>0$ and $\omega>0$ for $\zeta \in[0,1]$, and

$$
\begin{aligned}
\|u\|=\max _{\zeta \in[0,1]}|u(\zeta)| & =\max _{\zeta \in[0,1]} \int_{0}^{1} G_{1 \alpha}(\zeta, \tau) a(\tau) f(\tau, v(\tau), v(\tau)) d \tau \\
& \leq \int_{0}^{1} G_{1 \alpha}(\tau) a(\tau) f(\tau, v(\tau), v(\tau)) d \tau \\
& <\int_{0}^{1} G_{1 \alpha}(\tau) a(\tau) \frac{\|v\|}{\sigma_{1}} d \tau \\
& =\frac{1}{\sigma_{1}} \int_{0}^{1} G_{1 \alpha}(\tau) a(\tau) d \tau\|v\| \\
& =\|v\| .
\end{aligned}
$$

Similarly, $\|v\|<\|u\|,\|v\|<\|\omega\|$ and $\|\omega\|<\|v\|$, which is a contradiction, and Theorem is received.

Theorem 1.19. Assume that $\left(B_{1}\right)-\left(B_{3}\right)$ hold, and

$$
\begin{aligned}
f(\zeta, u, u) & >\frac{u}{\Upsilon_{\alpha}^{2} \int_{\theta}^{1-\theta} G_{1 \alpha}(\tau) a(\tau) d \tau}, \\
g(\zeta, v, v) & >\frac{v}{\Upsilon_{\beta}^{2} \int_{\theta}^{1-\theta} G_{1 \beta}(\tau) b(\tau) d \tau}, \\
h(\zeta, \omega, \omega) & >\frac{\omega}{\Upsilon_{\gamma}^{2} \int_{\theta}^{1-\theta} G_{1 \gamma}(\tau) c(\tau) d \tau},
\end{aligned}
$$

for all $t \in[0,1], u>0, v>0, \omega>0$, then boundary value problem (2) has no positive solution.

Example 1.20. Consider the system of nonlinear fractional differential equations:

$$
\left\{\begin{array}{l}
D^{\frac{5}{3}} u(\tau)+\frac{\tau}{1+\tau}|\operatorname{sinv}(\tau)|=0, D^{\frac{3}{2}} v(\tau)+\frac{\tau}{1+\tau}|\sin \omega(\tau)|=0, D^{\frac{4}{3}} \omega(\tau) \\
+\frac{\tau}{1+\tau}|\sin u(\tau)|=0, \quad 0<\tau<1 \\
u(0)=0, u(1)=\int_{0}^{1} \tau u(\tau) d \tau, v(0)=0, v(1)=\int_{0}^{1} \tau v(\tau) d \tau, \omega(0)=0 \\
\omega(1)=\int_{0}^{1} \tau \omega(\tau) d \tau
\end{array}\right.
$$

Set $e(\tau), f(\tau), g(\tau) \in[0,+\infty)$ and $\tau \in[0,1]$, then we have

$$
\begin{aligned}
& \left|\frac{\tau}{1+\tau}\right| \operatorname{sine}(\tau)\left|-\frac{\tau}{1+\tau}\right| \operatorname{sinf}(\tau)|| \leq \frac{\tau}{1+\tau}|e(\tau)-f(\tau)|, \\
& \left|\frac{\tau}{1+\tau}\right| \sin f(\tau)\left|-\frac{\tau}{1+\tau}\right| \operatorname{sing}(\tau)|| \leq \frac{\tau}{1+\tau}|f(\tau)-g(\tau)|, \\
& \left|\frac{\tau}{1+\tau}\right| \operatorname{sing}(\tau)\left|-\frac{\tau}{1+\tau}\right| \operatorname{sine}(\tau)|| \leq \frac{\tau}{1+\tau}|g(\tau)-e(\tau)| .
\end{aligned}
$$


Therefore,

$$
\begin{gathered}
\rho=\int_{0}^{1} G_{1 \alpha}(\tau) a(\tau) m(\tau) d \tau \leq \int_{0}^{1} G_{1 \alpha}(\tau) d \tau, \\
\theta=\int_{0}^{1} G_{1 \beta}(\tau) b(\tau) k(\tau) d \tau \leq \int_{0}^{1} G_{1 \beta}(\tau) d \tau, \\
\kappa=\int_{0}^{1} G_{1 \gamma}(\tau) c(\tau) n(\tau) d \tau \leq \int_{0}^{1} G_{1 \gamma}(\tau) d \tau .
\end{gathered}
$$

With the use of Theorem 1.4, B.V.P (19) has a unique positive solution.

Example 1.21. Consider the system of nonlinear fractional differential equations:

$$
\left\{\begin{array}{l}
D^{\frac{5}{3}} u(\tau)+[v(\tau)]^{a}=0, D^{\frac{5}{3}} v(\tau)+[\omega(\tau)]^{b}=0, D^{\frac{5}{3}} \omega(\tau)+[u(\tau)]^{c}=0 \\
0<\tau<1 \\
u(0)=0, u(1)=\int_{0}^{1} \tau u(\tau) d \tau, v(0)=0, v(1)=\int_{0}^{1} \tau v(\tau) d \tau, \omega(0)=0 \\
\omega(1)=\int_{0}^{1} \tau \omega(\tau) d \tau
\end{array}\right.
$$

Let $f(\tau, v, v)=v a, g(\tau, u, u)=u b$ and $h(\tau, \omega, \omega)=\omega c, 0<a, b, c<1$. It is easy to see that $\left(B_{1}\right)-\left(B_{3}\right)$ hold. By simple computation, we have $f_{0}=g_{0}=h_{0}=\infty$ and $f^{\infty}=g^{\infty}=h^{\infty}=0$. Thus it follows that problem 20 has a positive solution by $\left(H_{1}\right)$.

Example 1.22. Consider the system of nonlinear fractional differential equations:

$$
\left\{\begin{array}{l}
D^{\frac{3}{2}} u(\tau)+[v(\tau)]^{a^{\prime}}=0, D^{\frac{3}{2}} v(\tau)+[\omega(\tau)]^{b^{\prime}}=0, D^{\frac{3}{2}} \omega(\tau)+[u(\tau)]^{c^{\prime}}=0, \\
0<\tau<1, \\
u(0)=0, u(1)=\int_{0}^{1} \tau u(\tau) d \tau, v(0)=0, v(1)=\int_{0}^{1} \tau v(\tau) d \tau, \omega(0)=0, \\
\omega(1)=\int_{0}^{1} \tau \omega(\tau) d \tau .
\end{array}\right.
$$

Let $f(\tau, v, v)=v a^{\prime}, g(\tau, u, u)=u b^{\prime}$ and $h(\tau, \omega, \omega)=\omega c^{\prime}, 0<a^{\prime}, b^{\prime}, c^{\prime}<1$. It is easy to see that $\left(B_{1}\right)-\left(B_{3}\right)$ hold. By simple computation, we have $f^{0}=g^{0}=h^{0}=0$ and $f_{\infty}=g_{\infty}=h_{\infty}=\infty$. Thus it follows that problem 21] has a positive solution by $\left(H_{3}\right)$.

\section{References}

[1] M. S. ABDO, Further results on the existence of solutions for generalized fractional quadratic functional integral equations, Journal of Mathematical Analysis and Modeling, (2020)1(1) : 33-46, doi:10.48185/jmam.v1i1.2.

[2] B. Ahmad, J. Nieto, Existence results for a coupled system of nonlinear fractional differential equations with three-point boundary conditions, Comput. Math. Appl. 58 (2009) 1838-1843.

[3] H. Afshari, M. Atapour, E. Karapınar, A discussion on a generalized Geraghty multi-valued mappings and applications. Adv. Differ. Equ. 2020, 356 (2020).

[4] H. Afshari, D. Baleanu, Applications of some fixed point theorems for fractional differential equations with Mittag-Leffler kernel, Advances in Difference Equations, 140 (2020), Doi:10.1186/s13662-020-02592-2.

[5] H. Afshari, S. Kalantari, D. Baleanu, Solution of fractional differential equations via $\alpha-\phi$-Geraghty type mappings. Adv. Differ. Equ. 2018, 347(2018), https://doi.org/10.1186/s13662-018-1807-4.

[6] H. Afshari, Solution of fractional differential equations in quasi-b-metric and b-metric-like spaces, Adv. Differ. Equ. 2018, 285(2018), https://doi.org/10.1186/s13662-019-2227-9.

[7] H. Afshari, M. Sajjadmanesh, D. Baleanu, Existence and uniqueness of positive solutions for a new class of coupled system via fractional derivatives. Advances in Difference Equations. 2020 Dec;2020(1):1-8, https://doi.org/10.1186/s13662-020-02568-2.

[8] H. Afshari, F. Jarad, and T., Abdeljawad, On a new fixed point theorem with an application on a coupled system of fractional differential equations. Advances in Difference Equations 2020.1 (2020): 1-13, https://doi.org/10.1186/s13662-020-02926-0.

[9] H. Aydi, E. Karapınar, W. Shatanawi, Tripled fixed point results in generalized metric spaces. J. Appl. Math. 10 (2012). Article ID 314279. 
[10] E. Karapınar, Couple fixed point theorems for nonlinear contractions in cone metric spaces Computers and Mathematics With Applications Volume: 59 Issue: 12 Pages: 3656-3668 Published: JUN 2010.

[11] E. Karapinar, Fixed point theorems in cone Banach spaces, Fixed Point Theory Appl, (2009):9.

[12] E. Karapınar, H.D. Binh, N.H. Luc, and N.H., Can, On continuity of the fractional derivative of the time-fractional semilinear pseudo-parabolic systems, Advances in Difference Equations 2021, no. 1 (2021): 1-24.

[13] E. Karapınar, S.I. Moustafa, A. Shehata, R.P. Agarwal, Fractional Hybrid Differential Equations and Coupled Fixed-Point Results for $\alpha$-Admissible $F\left(\psi_{1}, \psi_{1}\right)$-Contractions in $M$-Metric Spaces, Discrete Dynamics in Nature and Society, Volume 2020, Article ID 7126045, 13 pages https://doi.org/10.1155/2020/7126045,2020.

[14] C. Li, X. Luo, Y. Zhou, Existence of positive solutions of the boundary value problem for nonlinear fractional differential equations, Comput. Math. Appl. 59 (2010) 1363-1375.

[15] H.R. Marasi, H. Afshari, M. Daneshbastam, C.B. Zhai, Fixed points of mixed monotone operators for existence and uniqueness of nonlinear fractional differential equations, Journal of Contemporary Mathematical Analysis, vol. 52, p. 8C13, (2017).

[16] S. Zhang, Positive solutions to singular boundary value problem for nonlinear fractional differential equation, Comput. Math. Appl. 59 (2010) 1300-1309.

[17] Y. Zhao, et al., Positive solutions for boundary value problems of nonlinear fractional differential equations, Appl. Math. Comput. 217 (2011) 6950-6958.

[18] V. Daftardar-Gejji, Positive solutions of a system of non-autonomous fractional differential equations, J. Math. Anal. Appl. $302(2005) 56-64$.

[19] J. Henderson, et al., Positive solutions for systems of generalized three-point nonlinear boundary value problems, Comment. Math. Univ. Carolin. 49 (2008) 79-91.

[20] C. Goodrich, Existence of a positive solution to a class of fractional differential equations, Appl. Math. Lett. 23 (2010) $1050-1055$.

[21] H. Salem, On the existence of continuous solutions for a singular system of nonlinear fractional differential equations, Appl. Math. Comput. 198 (2008) 445-452.

[22] X. Su, Existence of solution of boundary value problem for coupled system of fractional differential equations, Engrg. Math. 26 (2009) 134-137.

[23] C. Bai, J. Fang, The existence of a positive solution for a singular coupled system of nonlinear fractional differential equations, Appl. Math. Comput. 150 (2004) 611-621.

[24] M. Rehman, R. Khan, A note on boundary value problems for a coupled system of fractional differential equations, Comput. Math. Appl. 61 (2011) 2630-2637.

[25] W. Feng, et al., Existence of solutions for a singular system of nonlinear fractional differential equations, Comput. Math. Appl. 62 (2011) 1370-1378.

[26] H. Shojaat, H. Afshari, M.S. Asgari, A new class of mixed monotone operators with concavity and applications to fractional differential equations, TWMS J. App. and Eng. Math. V.11, N.1, 2021, pp. 122-133.

[27] X. Su, Boundaryvalue problem for a coupled system of nonlinear fractional differential equations, Appl. Math. Lett. 22 (2009) 64-69.

[28] A.A., Kilbas, H.M., Srivastava, j.j., Trujillo, (2006), Theory and applications of fractiona differential equations, NorthHolland Mathematics Studies. 204(204) 7-10.

[29] Podlubny, I. (1999), Fractional Differential Equations, Academic Press, New york.

[30] J. Wang, H. Xiang, Z. Liu, Positive solution to nonzero boundary values problem for a coupled system of nonlinear fractional differential equations, Internat. J. Differ. Equ. 2010 (2010) 12. Article ID 186928.

[31] W. Yang, Positive solutions for a coupled system of nonlinear fractional differential equations with integral boundary conditions, Computers and Mathematics with Applications 63 (2012) 288-297.

[32] E. Zeidler, Nonlinear Functional Analysis and Its Applications-I: Fixed-Point Theorems, Springer, New York, NY, USA, 1986.

[33] D. Guo, V. Lakshmikantham, X. Liu, Nonlinear Integral Equations in Abstract Spaces, in: Mathematics and Its Applications, vol. 373, Kluwer Academic Publishers, Dordrecht, The Netherlands, 1996. 